\title{
Predicting the Yield of Spring Maize Based on an Optimized Wavelet Neural Network with an Improved Double-Chain Quantum Genetic Algorithm
}

\author{
Wang bai ${ }^{1,2}$, Li Fanghua ${ }^{1}$, Huang Yan $^{1}$ and Meng Yan $^{1}$ \\ ${ }^{1}$ Heilongjiang Water Conservancy Institute, Harbin 150080, China, \\ ${ }^{2}$ Northeast Agricultural University, Harbin 150030, China \\ wangbai1980@163.com
}

\begin{abstract}
To overcome the shortcomings of the traditional wavelet neural network, an improved double-chain quantum genetic algorithm is used to optimize its parameters. This paper presents a prediction model for the optimized wavelet neural network that is applied to spring maize yields in the northeast of China. The results show that the coupled model is better than the traditional wavelet neural network, and achieves good prediction performance for the spring maize yield.
\end{abstract}

Keywords: Wavelet neural network; Quantum genetic algorithm; Spring maize yield

\section{Introduction}

Heilongjiang province is the largest grain producer in China, making it the most important commodity grain base in the country for assuring national food security ${ }^{[1][2]}$. The cultivated area of Heilongjiang province is about 200 million mu (approximately $133400 \mathrm{~km}^{2}$ ). Maize is one of the most important grain crops in Heilongjiang province, accounting for about $10 \%$ of the national maize planting area and directly affecting Chinese food safety[3]. Both irrigation and fertilization are preconditions for maize development in the region, although precipitation and water resources have a non-uniform space-time distribution. Thus, further expansion of the maize planting area could directly affect the stable production of maize. As such, growth indexes of maize have an important influence. Therefore, the accurate determination and prediction of maize yields has become an important basis for determining the irrigation schedule, water resource planning, and irrigation forecasting, which signal the sustainable development of water-saving agriculture.

Many scholars have developed prediction methods for maize yields. For example, Ji[4] used a logistic model to establish a corn tendency output sequence. The multiple regression forecast model and a revised disaster decrement rate yielded an accuracy of $90 \%$, providing the scientific basis for quantifying and refining the grain yield forecast. Wei[5] used statistical data from 2004-2013 in Shanxi Province, and employed exponential smoothing to predict the corn yield in future years. Su[6] applied the weight of each model in a combined forecasting model with entropy theory to forecast the corn yield. Zhang[7] used the grey $\operatorname{GM}(1,1)$ model to analyze regional experimental yields of corn varieties, and forecast the corn produced by Hebei province under conditions of relative stability. This model provided scientific information for production planning and decision making. Wang[8] used the well-calibrated and validated Agricultural Production Systems Simulator Model to assess spring maize potential yields at different levels, and identified the spatial-temporal characteristics of potential yield and yield gaps between different levels in Heilongjiang province from 1981-2010.

On the basis of the literature[9], this paper describes the improved Accelerating 
Double-Chain Quantum Genetic Algorithm (ADCQGA), which optimizes and compresses the search space of quantum chromosomes by changing the value of the quantum bits in the unit circle. We apply the improved ADCQGA to a wavelet neural network (WNN) to establish the ADCQGA-WNN model, and use this to predict the spring maize yield. egion.

\section{Improved Accelerating Double-Chain Quantum Genetic Algorithm}

\subsection{Structure and Principle of the Algorithm}

The original Double-Chain Quantum Genetic Algorithm ${ }^{[10][11][12]}$ (DCQGA) is a probability optimization algorithm based on the quantum calculation principle. DCQGA has the advantages of a small-scale population, rapid convergence, and good global search capacity, and is often applied to combinational optimization. In the literature ${ }^{[9]}$, the initial value $\theta_{i j}$ of probability amplitude of the quantum bit $\left[\cos \theta_{i j}, \sin \theta_{i j}\right]^{T}$ is randomly generated in the range $(0,2 \pi)$, and the quantum bit phase is updated using a quantum rotation gate. A quantum rotation gate is used to realize quantum mutation. The probability amplitudes of quantum bits, where $\left[\cos \theta_{i j}, \sin \theta_{i j}\right]^{T}$ is the periodic function in the process of evolution, are given by the argument $\theta_{i j}$ of the quantum chromosome phase. This is repeatedly located in the unit circle, so the search space of the quantum chromosomes is large, which prevents fast convergence. To improve the algorithm, we present an accelerated DCQGA in which the initial value $\theta_{i j}$ of the probability amplitude of the quantum bit is randomly generated in the range $(0, \pi / 2)$, which ensures good monotonicity in the fitness value of the population and the corresponding argument sorting, thus reducing the search space of the quantum chromosomes. The improved algorithm calculates the selection probability and expected reproductive rate based on the similarity and vector distance of quantum chromosomes in the search space, and optimizes and compresses the search space of the quantum chromosomes.

Algorithm Description:

The optimization problem in $n$-dimensional continuous space is expressed as follows:

$$
\left.\begin{array}{rl}
\min & f(x)=f\left(x_{1}, x_{2}, \cdots, x_{n}\right) \\
\text { s.t. } & a_{i} \leq x_{i} \leq b_{i} ; i=1,2, \cdots, n
\end{array}\right\}
$$

1) Population initialization. The initial chromosome population $Q(t)=\left\{q_{1}, q_{2}, \cdots q_{m}\right\}$ is randomly generated in the range $(0,2 \pi)$ by the DCQCA coding method, where $m$ is the population size, $n$ is the quantum digit capacity, $\theta_{0}$ is the initial step value of the angle, and $p_{m}$ is the mutation probability.

2) Solution space transformation. Every chromosome contains $2 n$ probability amplitudes of the quantum bit in the population, and these are mapped to the solution space of the continuous optimization problem in equation (1) using a linear transform. The $i$ th quantum bit of quantum chromosome $q_{j}$ is $\left[\alpha_{i}^{j}, \beta_{i}^{j}\right]^{T}$, and the variables of the corresponding solution space are:

$$
\begin{aligned}
& X_{i c}^{j}=a_{i}+\alpha_{i}^{j}\left(b_{i}-a_{i}\right) \\
& X_{i s}^{j}=a_{i}+\beta_{i}^{j}\left(b_{i}-a_{i}\right)
\end{aligned}
$$

Where $X_{i c}^{j}$ expresses the cosine solution of the $i$ th quantum bit for chromosome $j$ and $X_{i s}^{j}$ expresses the sine solution of the $i$ th quantum bit for chromosome $j$, $i=1,2, \cdots, m, \quad j=1,2, \cdots, n$.

3) Calculation and evaluation of the expected reproductive rate and selection probability based on the vector distance of chromosomes in population $Q(t)$. The $m$ chromosomes constitute a nonempty immune system $X$. The vector distance between 
chromosomes in set $\quad X$ is regulated as follows:

$$
\rho\left(x_{i}\right)=\sum_{j=1}^{m} f\left(x_{i}\right)-f\left(x_{j}\right)
$$

The density of quantum chromosomes is:

$$
\text { Densit }\left(x_{i}\right)=\frac{1}{\rho\left(x_{i}\right)}=\frac{1}{\sum_{j=1}^{m} f\left(x_{i}\right)-f\left(x_{j}\right)}
$$

The probability option formula of quantum chromosomes is given by:

$$
P\left(x_{i}\right)=\frac{\rho\left(x_{i}\right)}{\sum_{i=1}^{m} \rho\left(x_{i}\right)}=\frac{\sum_{j=1}^{m}\left|f\left(x_{i}\right)-f\left(x_{j}\right)\right|}{\sum_{i=1}^{m} \sum_{j=1}^{m}\left|f\left(x_{i}\right)-f\left(x_{j}\right)\right|}
$$

and the expected reproductive rate $e\left(x_{i}\right)$ of quantum chromosomes $f\left(x_{i}\right)$ is:

$$
e\left(x_{i}\right)=\frac{f\left(x_{i}\right)}{C\left(x_{i}\right)}=\sum_{k=1}^{m} f\left(x_{i}\right)\left|f\left(x_{i}\right)-f\left(x_{k}\right)\right|
$$

where $i, j, k=1,2, \cdots, m$.

4) According to the expected reproductive rate of quantum chromosomes, the algorithm makes $u$ of the quantum chromosomes in population $Q(t)$ into the new population $P(t)$.

5) On the basis of the selection probability, the quantum bits of $v$ quantum chromosomes are subjected to the rotation gate updating and mutation operation of DCQCA. These chromosomes are selected with a large selection probability from population $Q(t)$ and entered into the new population $P(t)$. The quantitative relation of these quantum chromosomes should satisfy $u+v=m$.

6) The fitness values of population $P(t)$ are evaluated by an objective function, and the best quantum chromosome and its corresponding quantum bit coding are recorded.

7) Optimize and compress the search space of quantum bit argument $\theta$. The angles of the quantum bit argument $\theta_{i j}$ are sorted for all quantum chromosomes in $P(t)$, and the maximum $\theta_{i j \max }$ and minimum $\theta_{i j \min }$ are recorded. To improve the flexibility and robustness of the algorithm, the parameter $\varepsilon$ is used to set the search space of argument $\theta_{i j}$ such that $\left|\theta_{i j \max }-\theta_{i j \min }\right|<\varepsilon$.

8) Accelerating the iterative process. If the convergence condition or number of iterations does not satisfy the termination requirement, the interval $\left(\theta_{i j \min }, \theta_{i j \max }\right)$ of the optimized argument $\theta_{i j}$ is used as the new initial variable interval to generate a new random population $Q(t)$ with $m$ members and $n$ quantum bits, and the algorithm returns to step (2).

\subsection{Simulation Comparison}

To verify the advantages and effectiveness of ADCQGA, Shaffer's F6 function was used to solve the extremum optimization problem using both ADCQGA and DCQGA ${ }^{[9]}$. Shaffer's F6 function can be written as:

$$
f(x, y)=0.5-\frac{\sin ^{2} \sqrt{x^{2}+y^{2}}-0.5}{\left(1+0.001\left(x^{2}+y^{2}\right)\right)^{2}}
$$

The function has infinite local maxima and a global maximum of 1 at the point $(0,0)$, and the value range of independent variables is $(-100,100)$.

ADCQGA was executed 10 times with a population size of $m=100$, quantum bits $n=2$, selection probability $P_{r}=0.009$, mutation probability $P_{m}=0.05$, initial value of angle step $\theta_{0}=0.05 \pi$, number of iterations $L_{\max }=300$, and $\varepsilon=10^{-4}$. The results of this 
comparison are presented in Table 1.

Table 1. Comparison of Optimization Results for the F6 Function Extremum (10 Experiments)

\begin{tabular}{crcccc}
\hline Algorithm & \multicolumn{5}{c}{ Shaffer's F6 function } \\
\hline & Iteration & Optimum result & $\begin{array}{c}\text { Poorest } \\
\text { result }\end{array}$ & $\begin{array}{c}\text { Average } \\
\text { result }\end{array}$ & $\begin{array}{c}\text { Convergenc } \\
\text { e times }\end{array}$ \\
DCQGA & 500 & 0.9993 & 0.99028 & 0.9958 & 9 \\
ADCQGA & 300 & 0.9998 & 0.9958 & 0.9979 & 10 \\
\hline
\end{tabular}

ADCQGA achieved an average of 0.9979 over 10 experiments, requiring an average of 56 iterations. The optimal performance of ADCQGA was superior to that of DCQGA in terms of both quality and efficiency. Thus, the results indicate that the improved strategy is effective and feasible.

\section{WNN Prediction Model based on ADCQGA}

\subsection{Principle and Structure of WNN}

Wavelet neural networks[13][14][15][16] are a kind of feed-forward neural network combined with wavelet analysis. WNNs use the wavelet function to replace the traditional S-shaped activation function, thus making full use of the wavelet transform and the self-learning ability of an artificial neural network. The proposed method uses a three-layer network with only one hidden layer. The hidden layer uses the wavelet function as the activation function, and the neurons of the output layer employ a sigmoid activation function.

Let $m$ be the number of samples, $p$ be the number of input layer nodes, $n$ be the number of hidden layer nodes, $q$ be the number of output layer nodes, $w_{i j}$ be the weight of the connection between hidden layer nodes and output layer nodes, $w_{j k}$ be the weight of the connection between hidden layer nodes and input layer nodes, $\theta_{j}$ be the threshold value of hidden layer nodes, $\theta_{i}$ be the threshold value of output layer nodes, and $a_{j}, b_{j}$ be the expansion coefficient and translation coefficient, respectively. The WNN is expressed as follows:

$$
y_{m}^{i}=f\left(\sum_{j=1}^{n} w_{i j} \psi_{a, b}\left(\sum_{k=1}^{p} w_{j k} x_{m}^{k}\right)\right)
$$

The sigmoid function can be written as:

$$
f(x)=1 /[1+\exp (-x)]
$$

To ensure strong robustness, small errors, and computational stability, we use the Morlet mother wavelet:

$$
\psi(x)=\cos (1.75 x) \exp \left(-x^{2} / 2\right)
$$

For the output of a single node, the mean square error (MSE) of the network output is calculated as:

$$
M S E=\frac{1}{2} \sum_{m=1}^{m}\left(y_{m}-d_{m}\right)^{2}
$$

The gradient descent method is used to train the network. By adjusting and optimizing the weights, threshold value, expansion coefficient, and translation coefficient of the network, the final MSE between the calculated output and expected output is minimized.

Adjustment of network parameters: 


$$
\begin{gathered}
n e t_{m}^{j}=\sum_{k=0}^{m} w_{j k} x_{m}^{k} \\
\psi_{a, b}\left(n e t_{m}^{j}\right)=\psi\left(\frac{n e t_{m}^{j}-b_{j}}{a_{j}}\right) \\
\delta_{w i}=-\sum_{m=1}^{m}\left(d_{m}^{i}-y_{m}^{i}\right) w_{i j} \psi_{a, b}\left(n e_{m}^{j} t\right) \\
\delta_{w_{j k}}=-\sum_{m=1 i=1}^{m} \sum_{i}^{q}\left(d_{m}^{i}-y_{m}^{i}\right) w_{i j} \psi_{a, b}^{\prime}\left(n e t_{m}^{j}\right) x_{m}^{k} / a_{j} \\
\delta_{b_{j}}=-\sum_{m=1 i=1}^{m} \sum_{i=1}^{q}\left(d_{m}^{i}-y_{m}^{i}\right) w_{i j} \psi_{a, b}^{\prime}\left(n e t_{m}^{j}\right)\left(\frac{n e t_{m}^{j}-b_{j}}{a_{j}}\right) / a_{j}
\end{gathered}
$$

After adjustment network parameters:

$$
\begin{gathered}
w_{i j}(n+1)=w_{i j}(n)-\eta \delta_{w_{i j}}+m c \Delta w_{i j}(n) \\
w_{j k}(n+1)=w_{j k}(n)-\eta \delta_{w_{j k}}+m c \Delta w_{j k}(n) \\
b_{j}(n+1)=b(n)-\eta b_{j}+m c \Delta b_{j}(n) \\
a_{j}(n+1)=a_{j}(n)-\eta \delta_{a_{j}}+m c \Delta b_{j}(n)
\end{gathered}
$$

Network parameters are repeatedly adjusted, when termination condition is meeting, the predicted results are outputted. The $m c$ is momentum factor.

\subsection{Realization of ADCQGA-WNN}

Traditional WNNs use the gradient descent method for parameter optimization. As a result they easily fall into local minima and suffer from slow convergence speeds ${ }^{[18][19]}$. ADCQCA is a highly efficient and robust global optimization algorithm. Optimal values of the weights, threshold value, expansion and translation coefficient are obtained by the improved ADCQCA instead of the random assignment of network initial parameters, thus overcoming the problem of error propagation. The optimized WNN parameters are further trained by the gradient descent method, and the twice-optimized results are regarded as the final WNN parameters. The ADCQGA-WNN parameters are trained by global optimization of ADCQCA and local optimization of WNN, thus improving both the speed and precision of the algorithm.

\subsection{Steps of ADCQCA-WNN}

(1) Determine the parameters of the algorithm. According to an input-output sample set, the network topology is determined and standardized, and the model takes the weight, threshold value, expansion and translation coefficient of the neural network as the quantum chromosome. Thus, every quantum chromosome contains two juxtaposed lines as its gene chain.

(2) Population initialization. The genetic chain of every quantum chromosome is expressed as $Q=\left\lfloor w_{i j}, w_{j k}, \theta_{j}, \theta_{k}, a_{j}, b_{j}\right\rfloor, \quad(i=1,2 \cdots, p), \quad(j=1,2 \cdots, n), \quad(k=1,2 \cdots, q)$. The total number of quantum chromosomes in the population is $N$, and there are $2 N$ genetic chains. All quantum chromosome genes $\left(\alpha_{i}, \beta_{i}\right) \quad(i=1,2 \cdots, m)$ are randomly generated in $[-3 / \sqrt{p}, 3 / \sqrt{p}]$.

(3) Fitness evaluation. Based on ADCQGA, the MSE of the WNN is taken as the evaluation index of the quantum population. Higher fitness values of the quantum chromosomes indicate a smaller error between the actual output and expected output. The best quantum chromosome and its corresponding quantum bit coding are recorded, and this quantum chromosome provides the optimized network parameters for the WNN on the next iteration. 
(4) Judging the termination condition. The objective is to judge whether the fitness value and iteration time have reached the maximal set value. When the conditions do not satisfy the above requirements, the quantum chromosomes are updated by ADCQGA. The algorithm generates a new quantum population, and returns to step (2). When the conditions satisfy the above requirements, the global optimal quantum chromosome of the WNN is used to define the weight, threshold value, expansion and translation coefficient of the WNN, namely the optimal solution. The WNN is established with the optimized parameters.

(5) Carrying on the operation of the WNN and generating the optimal solution. The above optimal solution is used to give the initial weight, threshold value, expansion and translation coefficient of the WNN, which is trained by gradient descent until the network reaches the prescriptive index value. Once the WNN based on ADCQGA has reached the best state in the training stage, the final network parameters are applied to forecast the maize yield in that state. The expected result is then exported for analysis.

\section{Application Example of ADCQGA-WNN}

\subsection{Application Example}

An experiment using integrated drip-irrigation and maize fertilizer was implemented at the test center of Heilongjiang Water Conservancy Institute in 2015. In the test center, the perennial mean temperature was set to $3.1^{\circ} \mathrm{C}$, the frostless period was $130-140 \mathrm{~d}$, the multi-year average precipitation was $400-650 \mathrm{~mm}$, and the soil texture was loamy. The average dry density was $1.34 \mathrm{~g} / \mathrm{cm}^{3}$, the field water-holding rate was $0.36 \mathrm{~cm}^{3} / \mathrm{cm}^{3}$, the organic matter content of the soil was $25.07 \mathrm{~g} / \mathrm{kg}$, and the average initial nitrate content was $67.79 \mathrm{mg} / \mathrm{kg}$. The nitrogen rate and irrigation quantity were selected as experimental factors. The nitrogen rate that did not contain base fertilizer, for each nitrogen split, four nitrogen of urea levels of $600,450,300$ and $0 \mathrm{~kg} \mathrm{~N} \mathrm{hm}-2$ were tested, the nitrogen application of urea splits at the jointing, heading and filling stages were used. For each irrigation split, two nitrogen of urea levels of $10 \mathrm{~mm}$ and $20 \mathrm{~mm}$ in the unit area were tested, the irrigation splits at the jointing, heading and filling stages were used.

The maize was sowed on May 12th and harvested in September. Using planting of one big ridge two rows with plastic film mulching. The distance between two adjacent ridges was $110 \mathrm{~cm}$, the row spacing of maize above ridge was $40 \mathrm{~cm}$, width of ridge was $90 \mathrm{~cm}$, the bottom width of ridge and furrow $30 \mathrm{~cm}$, the height of ridge was $15 \mathrm{~cm}$, the width of film was $120 \mathrm{~cm}$, the thickness of film was $0.008 \mathrm{~mm}$. Each experimental district had four ridges, which comprised two rows of maize.

There were eight experimental treatments, each of which was repeated three times to give a total of 24 experimental treatments. The size of each experimental treatment was 2 $\mathrm{m} \times 2.5 \mathrm{~m}$.

We considered 20 experimental treatments, and recorded the maize ear length, barren ear tip, 100-seed weight, leaf area index, chlorophyll relative content of maize plant, nitrogen application rate, and irrigation quantity as input variables. The maize yield was taken as the output variable. Four experimental treatments were used as test samples, and were not included in the aforementioned 20 experimental treatments. 


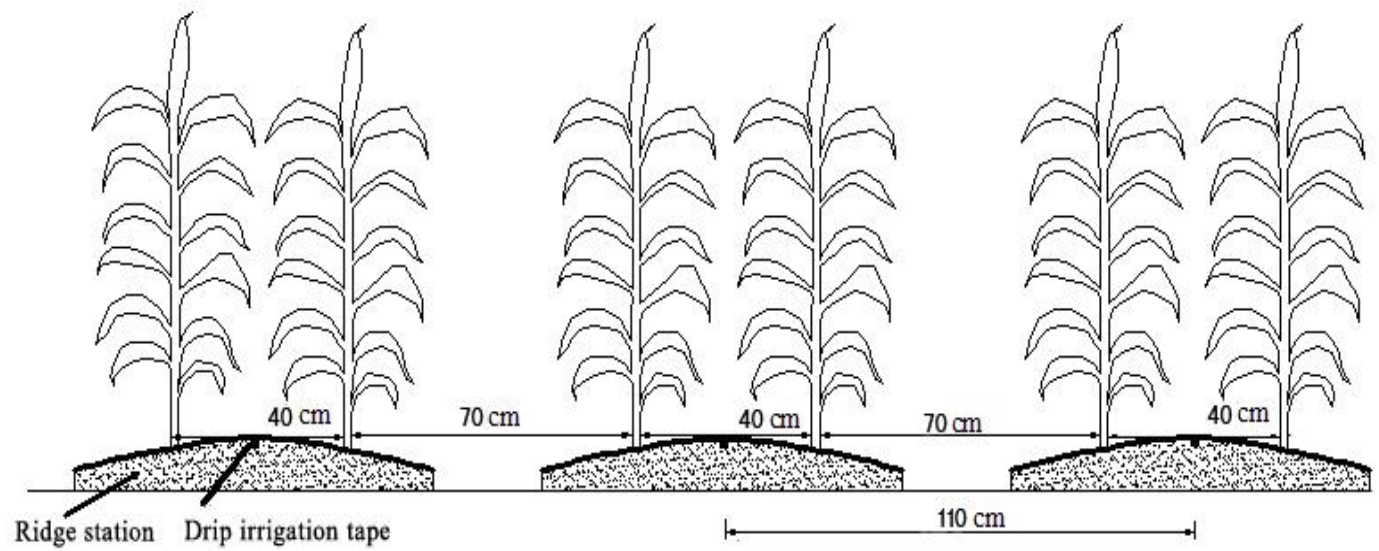

Figure 1. Planting Patterns of Mulched and Drip-irrigated Maize

Table 2. The Physical Properties of Soil

\begin{tabular}{cccccc}
\hline $\begin{array}{c}\text { Depth } \\
\text { /cm }\end{array}$ & \multicolumn{2}{c}{ The percentage of particles of different sizes } & & \multirow{2}{*}{$\begin{array}{c}\text { organic atter } \\
\text { content }\end{array}$} \\
\cline { 2 - 4 } & $2.0-0.02 \mathrm{~mm}$ & $0.02-0.002 \mathrm{~mm}$ & $<0.002 \mathrm{~mm}$ & & $\mathrm{mg} / \mathrm{kg}$ \\
\hline $0 \sim 20$ & 39.06 & 54.44 & 6.50 & silt loam soil & 31.63 \\
$20 \sim 40$ & 34.48 & 58.70 & 6.82 & silt loam soil & 25.50 \\
$40 \sim 80$ & 33.39 & 59.37 & 7.25 & silt loam soil & 18.07 \\
\hline
\end{tabular}

Table 3. The Experimental Scheme

\begin{tabular}{cccc}
\hline $\begin{array}{c}\text { Experiment } \\
\text { scheme }\end{array}$ & Base fertilizer & The nitrogen rate & Irrigation split \\
\hline 1 & 450 & 600 & $10 \mathrm{~mm}$ \\
2 & 450 & 600 & $20 \mathrm{~mm}$ \\
3 & 450 & 450 & $10 \mathrm{~mm}$ \\
4 & 450 & 450 & $20 \mathrm{~mm}$ \\
5 & 450 & 300 & $10 \mathrm{~mm}$ \\
6 & 450 & 300 & $20 \mathrm{~mm}$ \\
7 & 450 & 0 & $10 \mathrm{~mm}$ \\
8 & 450 & 0 & $20 \mathrm{~mm}$ \\
\hline
\end{tabular}

Table 4. The Measurement Indexes of Different Experiment Treatments for Simulation Data of the Model

\begin{tabular}{ccccccccc}
\hline Treatment & $\begin{array}{c}\text { Maize } \\
\text { Ear } \\
\text { Length }\end{array}$ & $\begin{array}{c}\text { Barren } \\
\text { ear tip }\end{array}$ & $\begin{array}{c}\text { The } \\
\text { 100-seed } \\
\text { weight }\end{array}$ & $\begin{array}{c}\text { Topdressing } \\
\text { fertilizer of } \\
\text { urea }\end{array}$ & $\begin{array}{c}\text { Irrigation } \\
\text { quantity }\end{array}$ & $\begin{array}{c}\text { Leaf } \\
\text { area } \\
\text { index }\end{array}$ & $\begin{array}{c}\text { Chlorophyll } \\
\text { relative } \\
\text { content }\end{array}$ & $\begin{array}{c}\text { Maize } \\
\text { yield }\end{array}$ \\
\cline { 2 - 9 } & $\mathrm{cm}$ & $\mathrm{cm}$ & $\mathrm{g}$ & $\mathrm{kg} / \mathrm{hm}-2$ & $\mathrm{~mm}$ & & & $\mathrm{t} / \mathrm{hm}-$ \\
2 & 19.05 & 0.23 & 35.39 & 300 & 30.00 & 4.73 & 52.67 & 14.19 \\
\hline 1 & 18.73 & 0.48 & 35.38 & 300 & 30.00 & 4.97 & 52.60 & 13.38 \\
3 & 18.26 & 0.58 & 35.82 & 300 & 30.00 & 5.21 & 52.58 & 13.93 \\
4 & 20.00 & 0.07 & 35.44 & 450 & 30.00 & 5.95 & 57.12 & 15.93 \\
5 & 19.83 & 0.17 & 36.04 & 450 & 30.00 & 5.23 & 60.46 & 14.75 \\
\hline
\end{tabular}




\begin{tabular}{ccccccccc}
\hline 6 & 19.78 & 0.28 & 36.42 & 600 & 30.00 & 4.93 & 65.12 & 14.86 \\
7 & 16.66 & 0.56 & 34.87 & 600 & 30.00 & 5.06 & 60.22 & 11.80 \\
8 & 18.37 & 0.68 & 35.94 & 300 & 60.00 & 5.20 & 58.14 & 13.79 \\
9 & 18.36 & 0.83 & 35.98 & 300 & 60.00 & 5.20 & 59.02 & 13.80 \\
10 & 19.62 & 0.93 & 36.83 & 300 & 60.00 & 6.04 & 59.84 & 15.72 \\
11 & 18.48 & 0.25 & 35.26 & 450 & 60.00 & 7.93 & 60.84 & 14.64 \\
12 & 19.55 & 0.05 & 39.26 & 450 & 60.00 & 5.10 & 58.81 & 15.63 \\
16 & 18.21 & 0.27 & 35.29 & 600 & 60.00 & 6.44 & 57.48 & 12.92 \\
14 & 20.27 & 0.09 & 35.10 & 600 & 60.00 & 5.43 & 65.40 & 15.47 \\
15 & 19.98 & 0.15 & 36.08 & 600 & 60.00 & 6.51 & 60.94 & 15.29 \\
16 & 18.93 & 0.62 & 34.25 & 0 & 30.00 & 4.97 & 51.55 & 12.33 \\
17 & 19.28 & 0.24 & 35.58 & 0 & 30.00 & 4.89 & 46.98 & 10.65 \\
18 & 18.55 & 0.38 & 33.19 & 0 & 60.00 & 4.89 & 44.96 & 13.50 \\
19 & 18.43 & 0.35 & 35.13 & 0 & 60.00 & 5.23 & 52.72 & 12.10 \\
20 & 19.15 & 0.35 & 35.68 & 0 & 60.00 & 5.53 & 51.10 & 12.36 \\
\hline
\end{tabular}

Table 5. The Measurement Indexes of Different Experiment Treatments for Testing Data

\begin{tabular}{|c|c|c|c|c|c|c|c|c|}
\hline $\begin{array}{c}\text { Treatme } \\
\text { nt }\end{array}$ & $\begin{array}{c}\text { Maize } \\
\text { Ear } \\
\text { Lengt } \\
\text { h } \\
\mathrm{cm}\end{array}$ & $\begin{array}{l}\text { Barre } \\
\text { near } \\
\text { tip }\end{array}$ & $\begin{array}{c}\text { The } \\
100 \text {-see } \\
\text { d weight }\end{array}$ & $\begin{array}{l}\text { Topdressin } \\
\mathrm{g} \text { fertilizer } \\
\text { of urea } \\
\mathrm{kg} / \mathrm{hm}-2\end{array}$ & $\begin{array}{l}\text { Irrigatio } \\
\text { n } \\
\text { quantity } \\
\text { mm }\end{array}$ & $\begin{array}{c}\text { Leaf } \\
\text { area } \\
\text { inde } \\
x \\
4.73\end{array}$ & $\begin{array}{c}\text { Chloroph } \\
\text { yll relative } \\
\text { content } \\
52.67\end{array}$ & $\begin{array}{c}\text { Maiz } \\
e \\
\text { yield } \\
\text { t/hm- } \\
2 \\
\end{array}$ \\
\hline 1 & 17.03 & 0.23 & 35.82 & 450 & 30.00 & 5.06 & 51.72 & 13.24 \\
\hline 2 & 19.06 & 0.35 & 36.95 & 600 & 30.00 & 5.20 & 54.41 & 14.57 \\
\hline 3 & 19.01 & 0.52 & 37.26 & 450 & 60.00 & 6.74 & 64.96 & 15.13 \\
\hline 4 & 18.53 & 1.02 & 33.71 & 0 & 30.00 & 4.93 & 51.13 & 13.53 \\
\hline
\end{tabular}

(1) Data processing. Let $m$ be the number of input samples, $x_{m}^{k}$ be the $p$ elements of the $m$ input samples, $y_{m}^{i}$ be the actual output, and $d_{m}^{i}$ be the expected output. To eliminate different effects caused by different dimensions and units of influential factors, a normalization process was applied to the input samples as follows:

$$
X_{i j}^{\prime}=\frac{0.9-0.1}{\max X_{i j}-\min X_{i j}} X_{i j}+\left(0.9-\frac{0.9-0.1}{\max X_{i j}-\min X_{i j}} X_{i j}\right)
$$

where $X_{i j}$ is the variable before normalization, $i$ denotes input sample, $j$ is the number of elements contained by the sample, $\max X_{i j}$ is the maximum value of $j$ in sample $i$, and $\min X_{i j}$ is the minimum value of $j$ in sample $i$.

(2) Determination of network structure and model parameters. There are seven nodes in the input layer, one node in the output layer, and 10 nodes in the hidden layer. With this 7-10-1 network structure, the precision of the predictions is 0.01 , and the model has a good fitting effect. The ADCQGA parameters were set as follows: population size $m=50$, quantum bits $n=91$, selection probability $P_{r}=0.009$, mutation probability $P_{m}=0.05$, initial angle step $\theta_{0}=0.05 \pi$, number of iterations $L_{\max }=20$, limiting factor $\varepsilon=10^{-4}$, and a total of 10 iterations were executed. 


\subsection{Prediction Results Analysis}

The ADCQGA-WNN model and a traditional WNN model were trained by standardized sample data until the network output reached the precision requirement and the error converged to the expected value.

For the four test samples in the Table 4, a comparative analysis of the results given by the ADCQGA-WNN model and traditional WNN model is shown in Table 5.

Table 6. Comparison of the Proposed Method and Traditional WNN using the four Test Datasets

\begin{tabular}{cccccc}
\hline & $\begin{array}{c}\text { Different growth } \\
\text { period }\end{array}$ & Treatment 1 & Treatment 2 & Treatment 3 & Treatment 4 \\
\hline Measured & & 13.24 & 14.57 & 15.13 & 13.53 \\
values & WNN & 13.40 & 14.14 & 14.33 & 12.91 \\
$\begin{array}{c}\text { Predictive } \\
\text { value }\end{array}$ & ADCQGA-WNN & 13.36 & 14.37 & 14.82 & 13.22 \\
Average & WNN & 111 & 67 & 207 & 244 \\
training steps & ADCQGA-WNN & 82 & 56 & 78 & 97 \\
Absolute error & WNN & 0.16 & -0.43 & -0.80 & -0.62 \\
& ADCQGA-WNN & 0.12 & -0.20 & -0.31 & -0.31 \\
\hline
\end{tabular}

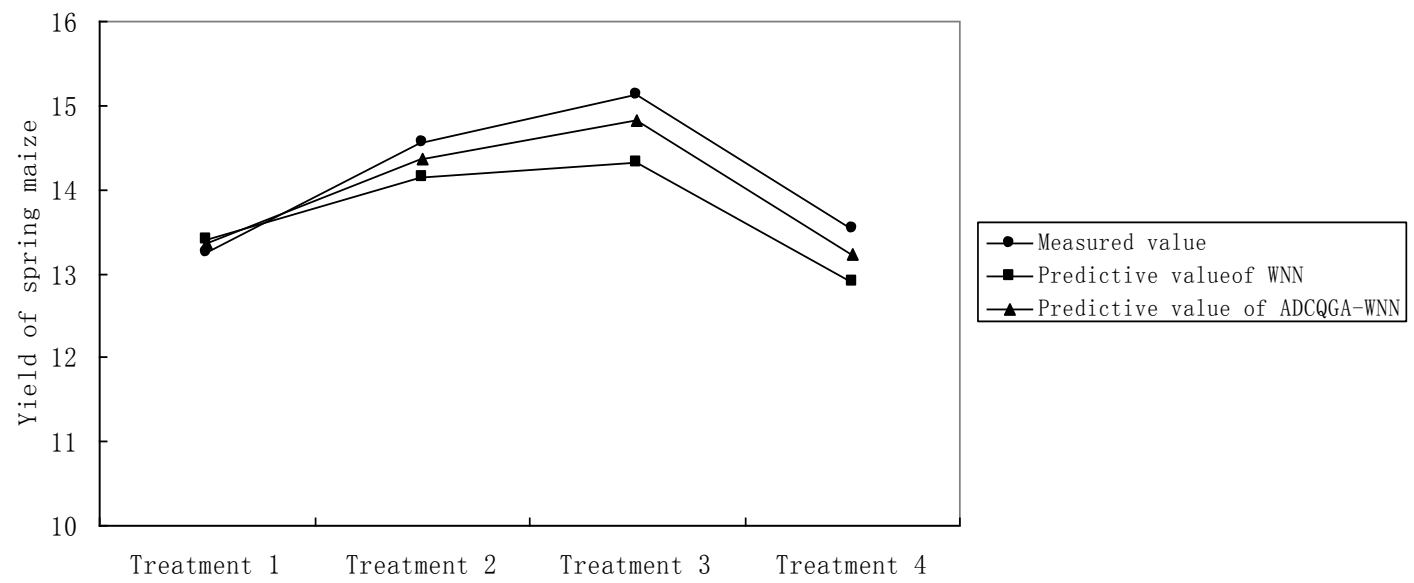

Figure 2. Fitting Chart of Measured Value and Predicted

The results show that the average number of training steps required by ADCQGA-WNN is fewer than that of traditional WNN, and the absolute error of ADCQGA-WNN is smaller than that of traditional WNN. The precision of the network prediction results with respect to the measured results is very good. Thus, the proposed model not only has higher accuracy, but also provides a theoretical basis for the production practice.

\section{Conclusions}

(1) In this paper, we have presented an improved Accelerating Double-Chain Quantum Genetic Algorithm. The algorithm ensures diversity in the quantum population, which avoids repetitive searching and redundant calculations by gradually optimizing and compressing the search space of the quantum chromosomes. This causes the population to 
accelerate toward the global optimal solution. On the premise of the excellent characteristics of DCQGA, the improved ADCQGA not only reduces the number of iterations and accelerates the convergence speed, but also improves the robustness and searching ability.

(2) The forecasting of the spring maize yield by the proposed ADCQGA-WNN was achieved by optimizing the weight, threshold value, expansion and translation coefficient of the WNN. The results show that the model has the good global optimization ability of quantum genetic algorithms and the fault-tolerance of WNNs. This leads to faster convergence and higher prediction precision than traditional WNNs, as well as good reliability for predicting the spring maize yield. The proposed model also provides a powerful theoretical basis for making a water-saving irrigation system for maize and food security.

\section{Acknowledgment}

The study was supported by the National Science and Technology Plan Projects (2014BAD12B01), Heilongjiang provincial Science Fund for Young Scholars (QC2015052), Heilongjiang postdoctoral Fund to pursue scientific research (LBH-Z14193).

\section{References}

[1] Liu Bo, Yang Xiaoguang, Wang Shigong. Calculation and Analysis of Climate Productive Potential of Major Grain Crops in Northeast China, Journal of Jilin Agricultural Sciences, 2012， 37(3): 57-60.

[2] Qiao Lei. Research on The Farmland Sustainable Utilization of Heilongjiang Province. TERRITORY \&NATURAL RESOURCES STUDY, 2012, (4): 16-17.

[3] Li Xiufen, Zhao Huiying, Zhu Haixia, et al. Evolution of maize climate productivity and its response to climate change in Heilongiiang Province, China[J]. Chinese Journal of Applied Ecology, 2016, (8).

[4] Ji Qi. Prediction of Maize Yield Based on Logistic and Disaster Decrement Rate Method. Chinese Agricultural Science Bulletin, 2012, 28(6): 293-296.

[5] Wei Jie, Ning Jing, Li Fuzhong. Forecasting of Corn Production in Shanxi Province-Based on the Exponential Smoothing Model. Tianjin Agricultural Sciences,2015,21(11): 84-85.

[6] Su Hengqiang, Chen Guifen, Zhu Chunrao. A Combination Forecasting Model for Corn Yield Based on Entropy Weight. Journal of Shenyang Agricultural University, 2010, 41(1): 125-127.

[7] Zhang Wenying, Li Qiusheng, Li Yuqin. Analysis and Forecast of Corn Yield of Hebei Province with Grey System [J]. Acta Agriculturae Boreali-Sinica, 2007, 22: 160-162.

[8] Wang Jing, Yang Xiaoguang, Lu Shuo, et al. Spatial-Temporal Characteristics of Potential Yields and Yield Gaps of Spring Maize in Heilongjiang Province. Scientia Agricultura Sinica, 2012, 45 (10): 1914-1925.

[9] Li Shiyong, Li Panchi. QUANTUM COMPUTATION AND QUANTUM OPTIMIZATION LGORITHMS, Harbin: Harbin institute of technology press, 2009: 69-84.

[10] Han K H, Kim J H. Quantum-inspired evolutionary algorithm for a class of combinational optimization. IEEE Transactions on Evolutionary Computing, 2002, 6(6):580-593.

[11] Li Xin, Cheng Chuntian, Zeng Yun. Training of process neural networks based on improved quantum genetic algorithm. Control and Decision, 2009, 24(3): 347-349.

[12] Gao Yinghui, Shen Zhenkang. An Angle-Coding Chromosome Quantum Genetic Algorithm. Computer Engineering \& Science, 2009, 31(3): 75-76.

[13] Huang Min, Cui Baotong, Gu Shusheng. Elevator Traffic Flow Prediction Based on Wavelet Neural Networks. Control and Decision, 2006, 21(5): 589-591.

[14] Cao Dayou. AN IMMUNE PARTICLE SWARM OPTIM IZATION AND ITS APPLICATION IN TRAINING WAVELET NEURAL NETWORKS. Computer Applications and Software，2009，26(6): 189-191.

[15] Chen Zhe, Feng Tianjin. RESEARCH ADVANCES ON COMBINATIN OF WZVELET ANALYSIS AND XEURAL NETWORKS. JOURNALOFELECTRONICS， 2000，22(3): 496-501.

[16] Mao Hongwei, Pan Hongxia, Liu Wenli. WAVELET NEURAL NETWORK BASED ON PARTICLE SWARM OPTIM IZATION ALGORITHM AND ITS APPLICATION IN FAULT DIAGNOSIS OF GEAR-BOX. JOURNAL OFVIBRATION AND SHOCK, 2007, 26(5): 133-134. 
[17] Li Jiejia, Li Shitao, Wang Lina. Application Research on Fault Prediction Based on Genetic Wavelet Neural Network. 2009, 25(2): 381-382.

[18] Li Jingyu, Li Qiqiang, Hou Haiyan, et, al. Traffic flow prediction based on the wavelet neural network with genetic algorithm. JOURNALOF SHANDONG UNIVERSITY (ENGINEERING SCIENCE) , 2007, 37(2): 109-111.

[19] Wang Shuo, Yang Shanlin, Ma Xijun. Nonlinear combination forecasting approach based on WNN-RAGA, 2008, (12): 160-162.

[20] Fu Qiang. System Analysis of Agricultural Water and Soil Resources and Comprehensive Evaluation. Beijing: China Water Resources and Hydropower Press, 2005: 126-128.

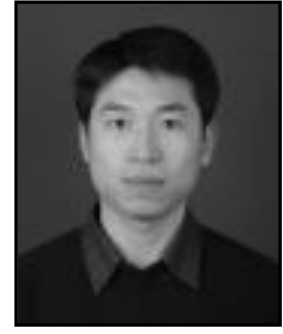

Bai Wang, male, born in 1980, is currently a grade senior engineer and a $\mathrm{PhD}$ in Heilongjiang Water Conservancy Institute, China. He received his $\mathrm{PhD}$ degree from Northeast Agricultural University, China, in 2013. His main research interests include theory and technology of water-saving irrigation. Email: wangbai1980@163.com. 
International Journal of Hybrid Information Technology

Vol. 10, No.2 (2017) 